\title{
Precision Constraints for Three-Flavor Neutrino Oscillations from the Full MINOS + and MINOS Dataset
}

P. Adamson, ${ }^{4}$ I. Anghel,${ }^{8}$ A. Aurisano, ${ }^{2}$ G. Barr, ${ }^{14}$ A. Blake, ${ }^{1,9}$ S. V. Cao, ${ }^{20}$ T. J. Carroll,${ }^{20,}$ C. M. Castromonte, ${ }^{5}$ R. Chen, ${ }^{11}$ S. Childress, ${ }^{4}$ J. A. B. Coelho, ${ }^{21}$ S. De Rijck, ${ }^{20}$ J. J. Evans, ${ }^{11}$ G. J. Feldman, ${ }^{6}$ W. Flanagan, ${ }^{3}$ M. Gabrielyan, ${ }^{12}$ S. Germani, ${ }^{10}$ R. A. Gomes, ${ }^{5}$ P. Gouffon, ${ }^{17}$ N. Graf,${ }^{15}$ K. Grzelak, ${ }^{22}$ A. Habig, ${ }^{13}$ S. R. Hahn, ${ }^{4}$ J. Hartnell, ${ }^{19}$ R. Hatcher, ${ }^{4}$ A. Holin, ${ }^{10}$ J. Huang, ${ }^{20}$ L. W. Koerner, ${ }^{7}$ M. Kordosky, ${ }^{23}$ A. Kreymer ${ }^{4}$ K. Lang, ${ }^{20}$ P. Lucas, ${ }^{4}$ W. A. Mann, ${ }^{21}$ M. L. Marshak, ${ }^{12}$ N. Mayer, ${ }^{21}$ R. Mehdiyev, ${ }^{20}$ J. R. Meier, ${ }^{12}$ W. H. Miller, ${ }^{12}$ G. Mills, ${ }^{24, *}$ D. Naples, ${ }^{15}$ J. K. Nelson, ${ }^{23}$ R. J. Nichol, ${ }^{10}$ J. O'Connor, ${ }^{10}$ R. B. Pahlka, Ž. Pavlović, ${ }^{4}$ G. Pawloski, ${ }^{12}$ A. Perch,${ }^{10}$ M. M. Pfützner, ${ }^{10}$ D. D. Phan,${ }^{20}$ R. K. Plunkett, ${ }^{4}$ N. Poonthottathil, ${ }^{4}$ X. Qiu, ${ }^{18}$ A. Radovic, ${ }^{23}$ P. Sail,${ }^{20}$ M. C. Sanchez,${ }^{8}$ J. Schneps,${ }^{21, \dagger}$ A. Schreckenberger ${ }^{20}$ R. Sharma, ${ }^{4}$ A. Sousa, ${ }^{2}$ N. Tagg, ${ }^{25}$ J. Thomas, ${ }^{10}$ M. A. Thomson, ${ }^{1}$ A. Timmons, ${ }^{11}$ J. Todd, ${ }^{2}$ S. C. Tognini, ${ }^{5}$ R. Toner, ${ }^{6}$ D. Torretta, ${ }^{4}$ P. Vahle, ${ }^{23}$ A. Weber, ${ }^{14,16}$ L. H. Whitehead, ${ }^{10}$ and S. G. Wojcicki ${ }^{18}$

(MINOS+ Collaboration)

\author{
${ }^{1}$ Cavendish Laboratory, University of Cambridge, Cambridge CB3 OHE, United Kingdom \\ ${ }^{2}$ Department of Physics, University of Cincinnati, Cincinnati, Ohio 45221, USA \\ ${ }^{3}$ Department of Physics, University of Dallas, Irving, Texas 75062, USA \\ ${ }^{4}$ Fermi National Accelerator Laboratory, Batavia, Illinois 60510, USA \\ ${ }^{5}$ Instituto de Física, Universidade Federal de Goiás, 74690-900, Goiánia, GO, Brazil \\ ${ }^{6}$ Department of Physics, Harvard University, Cambridge, Massachusetts 02138, USA \\ ${ }^{7}$ Department of Physics, University of Houston, Houston, Texas 77204, USA \\ ${ }^{8}$ Department of Physics and Astronomy, Iowa State University, Ames, Iowa 50011 USA \\ ${ }^{9}$ Lancaster University, Lancaster, LAI 4YB, United Kingdom \\ ${ }^{10}$ Department of Physics and Astronomy, University College London, \\ London WCIE 6BT, United Kingdom \\ ${ }^{11}$ Department of Physics and Astronomy, University of Manchester, Manchester M13 9PL, United Kingdom \\ ${ }^{12}$ University of Minnesota, Minneapolis, Minnesota 55455, USA \\ ${ }^{13}$ Department of Physics, University of Minnesota Duluth, Duluth, Minnesota 55812, USA \\ ${ }^{14}$ Subdepartment of Particle Physics, University of Oxford, Oxford OX1 3RH, United Kingdom \\ ${ }^{15}$ Department of Physics and Astronomy, University of Pittsburgh, Pittsburgh, Pennsylvania 15260, USA \\ ${ }^{16}$ Rutherford Appleton Laboratory, Science and Technology Facilities Council, \\ Didcot, OX11 OQX, United Kingdom \\ ${ }^{17}$ Instituto de Física, Universidade de São Paulo, CP 66318, 05315-970, São Paulo, SP, Brazil \\ ${ }^{18}$ Department of Physics, Stanford University, Stanford, California 94305, USA \\ ${ }^{19}$ Department of Physics and Astronomy, University of Sussex, \\ Falmer, Brighton BN1 9QH, United Kingdom \\ ${ }^{20}$ Department of Physics, University of Texas at Austin, Austin, Texas 78712, USA \\ ${ }^{21}$ Physics Department, Tufts University, Medford, Massachusetts 02155, USA \\ ${ }^{22}$ Department of Physics, University of Warsaw, PL-02-093 Warsaw, Poland \\ ${ }^{23}$ Department of Physics, College of William \& Mary, Williamsburg, Virginia 23187, USA \\ ${ }^{24}$ Los Alamos National Laboratory, Los Alamos, New Mexico 87545, USA \\ ${ }^{25}$ Otterbein University, Westerville, Ohio 43081, USA
}

(Received 30 June 2020; accepted 19 August 2020; published 21 September 2020)

We report the final measurement of the neutrino oscillation parameters $\Delta m_{32}^{2}$ and $\sin ^{2} \theta_{23}$ using all data from the MINOS and MINOS+ experiments. These data were collected using a total exposure of $23.76 \times 10^{20}$ protons on target producing $\nu_{\mu}$ and $\bar{\nu}_{\mu}$ beams and $60.75 \mathrm{kt} \mathrm{yr}$ exposure to atmospheric neutrinos. The measurement of the disappearance of $\nu_{\mu}$ and the appearance of $\nu_{e}$ events between the Near

\footnotetext{
Published by the American Physical Society under the terms of the Creative Commons Attribution 4.0 International license. Further distribution of this work must maintain attribution to the author(s) and the published article's title, journal citation, and DOI. Funded by $\operatorname{SCOAP}$.
} 
and Far detectors yields $\left|\Delta m_{32}^{2}\right|=2.40_{-0.09}^{+0.08}\left(2.45_{-0.08}^{+0.07}\right) \times 10^{-3} \mathrm{eV}^{2}$ and $\sin ^{2} \theta_{23}=0.43_{-0.04}^{+0.20}\left(0.42_{-0.03}^{+0.07}\right)$ at $68 \%$ C.L. for normal (inverted) hierarchy.

DOI: 10.1103/PhysRevLett.125.131802

Since the discovery of $\nu_{\mu}$ flavor disappearance oscillations in atmospheric neutrinos by the SuperKamiokande experiment in 1998 [1], determinations of neutrino masssquared differences and mixing angles have steadily improved [2-12], but the need for more precision remains. This is especially the case for the atmospheric $\theta_{23}$ mixing angle. A value corresponding to maximal mixing, $\theta_{23}=45^{\circ}$, may be a harbinger for an underlying symmetry. On the other hand, if the mixing is nonmaximal, determination of its octant is important for $\nu_{e}$-flavor appearance measurements and the inference of the $C P$ violating angle $\delta_{C P}[6]$.

This Letter reports new measurements of $\Delta m_{32}^{2}$ and $\sin ^{2}\left(\theta_{23}\right)$ using the complete set of beam and atmospheric data taken with the MINOS detectors. Two distinct beam energy configurations of the NuMI neutrino beam at Fermi National Accelerator Laboratory corresponded to two phases of the MINOS (2005-2012) and MINOS+ (2013-2016) long-baseline, on-axis neutrino oscillation experiments. The MINOS+ dataset significantly increases the statistics of the MINOS measurements [4] in the energy region above the oscillation maximum in the standard model of oscillations. Over this region extending from 1.5 to above $10 \mathrm{GeV}$ in $E_{\nu}$, MINOS and MINOS+ monitor the increase of $\nu_{\mu}$-flavor survival probability. This provides additional sensitivity, not available to narrow band beam experiments, for measuring the extent to which $\theta_{23}$ deviates from nonmaximal mixing. Monitoring this revival rate supplements the measurement of the depth of the oscillation maximum which occurs within a small span of $E_{\nu}$. Effects from nonstandard neutrino interactions [13], neutrino decay [14,15], decoherence [16], or the existence of sterile neutrinos $[17,18]$, could manifest themselves over the large energy range. Consequently, the measurements reported here will also allow a stringent test for such phenomena as well as future hypotheses, which lie outside the purview of conventional three-flavor neutrino oscillations.

The MINOS and MINOS+ long-baseline, on-axis neutrino oscillation experiments recorded two distinct phases of exposure to the NuMI neutrino beam [19] at Fermilab utilizing the MINOS Near and Far detectors [20]. Both detectors were functionally equivalent magnetized steelscintillator, tracking, sampling calorimeters. The Near Detector (ND) was $1.04 \mathrm{~km}$ from the target, $103 \mathrm{~m}$ underground, and had a mass of 980 t. The Far Detector (FD) was $735 \mathrm{~km}$ from the target, $705 \mathrm{~m}$ underground, and had a mass of $5.4 \mathrm{kt}$. The detectors had average toroidal magnetic fields of $1.4 \mathrm{~T}$, to enable the separation of $\nu_{\mu}$ from $\bar{\nu}_{\mu}$. The FD was also used to study atmospheric neutrinos [21] making use of the scintillator veto shield to improve cosmic muon background rejection.

During MINOS-phase data taking, the NuMI beam operated primarily in a low-energy beam configuration, producing muon neutrinos or antineutrinos, depending on the polarity of the pulsed magnetic horns, with a peak energy around $3 \mathrm{GeV}$. The MINOS low-energy beam exposure was $10.56 \times 10^{20}$ protons on target (POT) in $\nu_{\mu}$ mode and $3.36 \times 10^{20}$ POT in $\bar{\nu}_{\mu}$ mode. The MINOS $\nu_{\mu}$ mode sample included an additional $0.15 \times 10^{20}$ POT exposure in a high-energy $\nu_{\mu}$ mode with a peak $\nu_{\mu}$ energy of $9 \mathrm{GeV}$. The analysis of this MINOS-phase data has been described previously, presenting a $37.88 \mathrm{kt}$ yr sample of atmospheric neutrinos, measurements of both $\nu_{\mu}$ and $\bar{\nu}_{\mu}$ disappearance, and $\nu_{e}$ and $\bar{\nu}_{e}$ appearance [4,12,21,22].

This analysis uses the complete MINOS dataset described above and, in addition, includes $22.87 \mathrm{kt} \mathrm{yr}$ of atmospheric-neutrino data from 2011-2016, along with the complete three years of MINOS $+\nu_{\mu}$-mode beam data corresponding to an exposure of $9.69 \times 10^{20}$ POT. In the MINOS+ phase, the NuMI beam operated in the mediumenergy configuration, producing a $\nu_{\mu}$ beam peaking at near $7 \mathrm{GeV}$. The MINOS $+\nu_{\mu}$ charged current (CC) interactions in the ND were composed of $96.9 \% \nu_{\mu}, 1.9 \% \bar{\nu}_{\mu}$, and $1.2 \%$ $\left(\nu_{e}+\bar{\nu}_{e}\right)$. In comparison, the MINOS low-energy $\nu_{\mu}$-mode ND data were composed of $92.9 \% \nu_{\mu}, 5.8 \% \bar{\nu}_{\mu}$, and $1.3 \%$ $\left(\nu_{e}+\bar{\nu}_{e}\right) \mathrm{CC}$ interactions [23]. There is no $\nu_{e}$ appearance included in the MINOS+ phase analysis since the higher energy exposure increases the neutral-current (NC) backgrounds to the low energy $\nu_{e}$ appearance signal.

The Monte Carlo (MC) modeling was unchanged compared to the most recent previous publications. The accelerator beam neutrino flux was simulated using the FLUGG package [24] and the atmospheric neutrino flux using the Bartol calculations [25]. Beam and atmospheric neutrino interactions in the detector are simulated using NEUGEN3 [26], and interactions of atmospheric neutrinos in the surrounding rock are propagated into the detector using NUANCE [27]. The detector response to final-state particles is simulated, for both beam and atmospheric neutrino interactions, using a combination of GEANT3 [28] and GCALOR [29]. For MINOS+, the beam-neutrino reconstruction algorithms were tuned to account for the higher occupancy in the ND arising from the increased beam-neutrino flux. The data observed in the ND are used to tune the MINOS and MINOS+ flux simulations. The MINOS flux tuning procedure, described previously [23], was improved upon to separate the effect of the rate of 
secondary hadron production in the target from that of the charged particle focussing by the horns. This procedure combined data from special ND data taken with horn currents between 0 and $200 \mathrm{kA}$.

During the MINOS+ running period, it was observed that the neutrino energy peak position was shifted in the ND from that predicted by the MC by about $400 \mathrm{MeV}$. Furthermore, during the final running period, the upstream support of the first magnetic horn moved downward $4 \mathrm{~mm}$ over a period of a few months. Checks on these effects, however, showed that the oscillation parameter measurement is robust against these $\mathrm{MC}$ and data differences once the ND data are used to correct the neutrino flux to less than $0.05 \sigma$ in both oscillation parameters.

This analysis uses $\nu_{\mu}$ and $\bar{\nu}_{\mu}$ events, which result in a $\mu^{-}$ or $\mu^{+}$in the final state. Signal events have a characteristic muon track with a hadron shower near the interaction point. The major source of background is from $\mathrm{NC}$ events that produce hadron showers with short tracks. A multivariate $k$-nearest neighbor $(k-\mathrm{NN})$ algorithm [30] was used by MINOS to select CC $\nu_{\mu}$ and $\bar{\nu}_{\mu}$ interactions based on event topology and the characteristic muon track energy deposition. For MINOS+, the algorithm was trained using representative MINOS $+\mathrm{CC}$ and $\mathrm{NC}$ events from the $\mathrm{MC}$ [31]; the distribution of the $k$-NN discriminant in MINOS+ ND MC is shown in Fig. 1. Events with a value of the $k \mathrm{NN}$ discriminant below 0.3 were removed. The selected CC $\nu_{\mu}$ and $\bar{\nu}_{\mu}$ sample has a purity of $99.1 \%$ in the ND and $99.3 \%$ in the FD, with the impurities due to NC interactions.

The visible energy of the selected $\nu_{\mu}$ and $\bar{\nu}_{\mu}$ events was reconstructed from the sum of the muon track and the

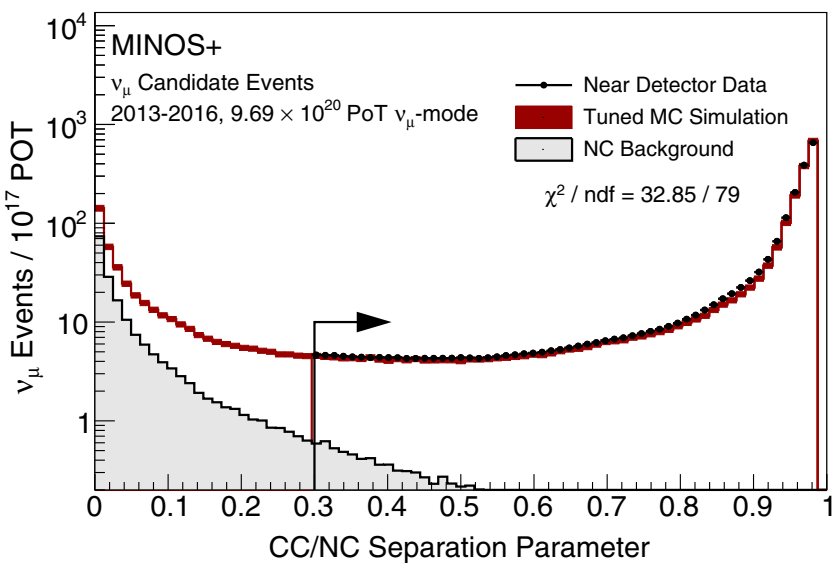

FIG. 1. The $k$-NN discriminant used in MINOS+ data to separate CC $\nu_{\mu}$ and $\bar{\nu}_{\mu}$ interactions from the background of $\mathrm{NC}$ interactions. The discriminant is shown for data and $\mathrm{MC}$ in the ND, where the MC has been corrected using the beam tuning described in the text. The red band is the systematic uncertainty on the MC prediction yielding a $\chi^{2} /$ d.o.f. of (32.85/79) for the data and MC comparison. Interactions in both detectors with the discriminant value above 0.3 are selected by the analysis. hadronic shower energy. The muon energy was measured from the range in the detector for tracks that were fully contained in the detector, or from the curvature in the magnetic field for tracks that exited the detector. The shower energy was estimated using another $k \mathrm{NN}$ algorithm that compares the topology of the event to a library of MC events and uses the closest-matching $\mathrm{MC}$ events to estimate the true energy of the hadron shower [31,32].

The beam data consist of $\nu_{\mu}$ and $\bar{\nu}_{\mu}$ events with reconstructed interaction vertices within the detector's fiducial volume. The MINOS $\nu_{\mu}$ dataset includes a sample of nonfiducial muons from neutrinos that interacted outside the detector's fiducial volume or in the rock surrounding the detector, identified by muons entering the front or sides of the detector in time with the beam. Since this sample has poorly reconstructed interaction energy, its impact on the oscillation measurement is limited and thus did not warrant selecting a similar sample from the MINOS+ data [33].

The MINOS + reconstructed $C C \nu_{\mu}$ and $\bar{\nu}_{\mu}$ energy spectra in the ND were used to predict the energy spectrum expected in the FD using a beam transfer matrix, as was done for MINOS [23]. This prediction for MINOS and MINOS+ combined without oscillations is shown in Fig. 2 (orange line) compared to the selected data (black points). Also shown is the ratio of observed FD events to the number of

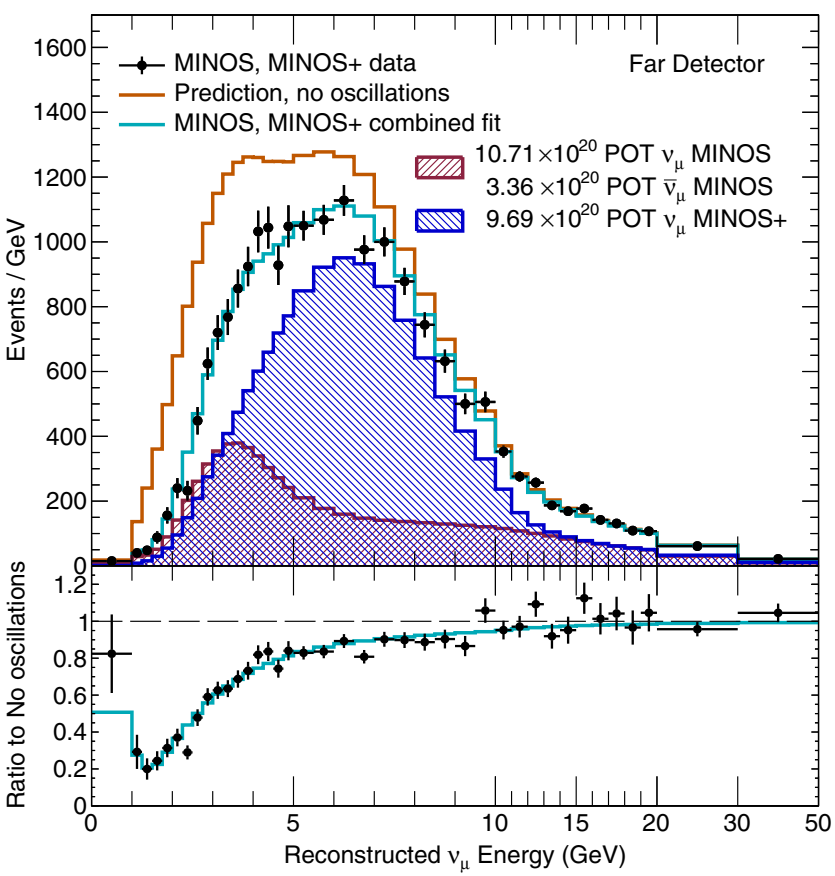

FIG. 2. Top: The reconstructed energy spectra for MINOS and MINOS+ events selected within the Far Detector fiducial volume for data (black points) adjusted for bin size, and the best fit MC predictions for MINOS (red hatched histogram), MINOS+ (blue hatched histogram) and the sum (cyan line). The prediction at the FD with no oscillations is shown as the orange line. Bottom: The ratios of the data and the oscillated prediction to the no oscillation prediction for MINOS and MINOS+ combined. 
predicted events assuming no oscillations as a function of reconstructed neutrino energy. The energy-dependent deficit of $\nu_{\mu}$ and $\bar{\nu}_{\mu}$ interactions is clearly observed, indicating the expected three-flavor oscillatory nature of the disappearance. The MINOS+ data provide significant additional statistical power integrated between 4 and $8 \mathrm{GeV}$ energy range over the lower energy MINOS data.

Atmospheric neutrinos were separated from the cosmic ray backgrounds into three separate samples [21,34-36]. The first sample of $\nu_{\mu}$ and $\bar{\nu}_{\mu} \mathrm{CC}$ interactions required a reconstructed interaction vertex in the detector's fiducial volume. The second sample were also contained-vertex, but showerlike events, primarily $\mathrm{CC} \nu_{e}, \mathrm{CC} \bar{\nu}_{e}$, and $\mathrm{NC}$ interactions that were used to constrain the atmospheric neutrino flux. The third sample required a reconstructed upward-going muon track and contained nonfiducial events that were initiated by atmospheric $\nu_{\mu}$ and $\bar{\nu}_{\mu}$ interactions that occurred in the rock around the detector. The number of observed and predicted neutrino events for MINOS and MINOS + are given in Table I.

The combined fit to the MINOS and MINOS $+\nu_{\mu}$ disappearance data was carried out independently of the MINOS $\nu_{e}$ appearance fit. To determine values of the oscillation parameters from the muon neutrino data, a maximum likelihood fit was performed by varying $\Delta m_{32}^{2}, \sin ^{2} \theta_{23}, \sin ^{2} \theta_{13}$, and $\delta_{C P}$ and using the negative log-likelihood function:

$$
-\ln \mathcal{L}=\sum_{j} \mu_{j}-n_{j}+n_{j} \ln \left(n_{j} / \mu_{j}\right)+0.5 \sum_{k}\left(\frac{\alpha_{k}}{\sigma_{\alpha_{k}}}\right)^{2},
$$

where $\mu_{j}$ and $n_{j}$ are the numbers of expected and observed events in bin $j$ of the reconstructed energy distribution, $\alpha_{k}$ include the fitted systematic parameters and a constraint on $\sin ^{2} \theta_{13}$ with corresponding uncertainties of $\sigma_{\alpha_{k}}$. The mixing angle $\theta_{13}$ was constrained to $\sin ^{2} \theta_{13}=0.0210 \pm$ 0.0011 [37]. The solar parameters were fixed to $\Delta m_{21}^{2}=$ $7.54 \times 10^{-5} \mathrm{eV}^{2}$ and $\sin ^{2} \theta_{12}=0.307$ [38] since they

TABLE I. Selected events in the Far Detector for all MINOS and MINOS + beam and atmospheric samples compared to no oscillations and to the best fit prediction.

\begin{tabular}{lcccrrr}
\hline \hline & \multicolumn{3}{c}{ Data } & & \multicolumn{2}{c}{ Predicted } \\
\cline { 2 - 4 } \cline { 7 - 8 } & MINOS & MINOS + & Total & & No Osc. & Best fit \\
\hline$\nu_{\mu}\left(\nu_{\mu}\right.$ beam $)$ & 2579 & 6280 & 8859 & & 10634 & 8851 \\
$\bar{\nu}_{\mu}\left(\nu_{\mu}\right.$ beam $)$ & 312 & 293 & 605 & & 677 & 598 \\
Nonfid. $\mu^{-}+\mu^{+}$ & 2911 & $\mathrm{~N} / \mathrm{A}$ & 2911 & & 3256 & 2838 \\
$\bar{\nu}_{\mu}\left(\bar{\nu}_{\mu}\right.$ beam $)$ & 226 & $\mathrm{~N} / \mathrm{A}$ & 226 & & 320 & 225 \\
Atm. $\nu_{\mu}+\bar{\nu}_{\mu}$ & 905 & 473 & 1378 & & 1885 & 1366 \\
Atm. $\mu^{-}+\mu^{+}$ & 466 & 270 & 736 & & 930 & 737 \\
Atm. showers & 701 & 422 & 1123 & & 1224 & 1130 \\
\hline \hline
\end{tabular}

have no effect on the oscillation parameter measurement. The likelihood function contained 17 nuisance parameters that accounted for the largest systematic uncertainties as discussed in previous publications [15,21].

Uncertainties on the flux of beam neutrinos were obtained from the fits performed using the ND data to tune the flux simulation. Separate uncertainties were calculated for the MINOS and MINOS+ beam-neutrino datasets. All uncertainties related to the interactions of neutrinos in the detector and neutrino reconstruction were unchanged from MINOS to MINOS+. All uncertainties on the atmospheric-neutrino samples were unchanged between the previously analyzed data and the new data added for this publication.

The effects of the systematic uncertainties on the $\nu_{\mu}$ disappearance measurement were studied with MC samples modified by shifting the uncertainties by one standard deviation. Table II shows the largest of the systematic uncertainties on the $\Delta m_{32}^{2}$ measurement. The dominant uncertainties associated with the beam data are the shower energy uncertainty and the relative normalization between the two detectors. The shower energy uncertainty, which has the second largest impact on $\Delta m_{32}^{2}$, averages at about $8 \%$ below $3 \mathrm{GeV}$ and approaches $6.6 \%$ at higher shower energies. The $1.6 \%$ relative normalization uncertainty accounts for differences in event selection and reconstruction between the ND and FD as well as uncertainties on each detector's fiducial mass and live time. The uncertainty on the measurement of the muon energy is fully correlated between the beam and atmospheric samples and is $2 \%(3 \%)$ when calculated from range and 3\% (5\%) when calculated from curvature [15] for the beam (atmospheric) samples. The difference between the samples is attributed to the orientation of the detector planes relative to the incident muons.

The $15 \%$ atmospheric normalization uncertainty for contained-vertex (CV) events comes from uncertainties on the flux and the neutrino cross section [21]. This normalization uncertainty has the largest effect on $\Delta m_{32}^{2}$. The atmospheric nonfiducial events (atm nonfid in Table II) have a normalization uncertainty of $25 \%$ due to larger flux uncertainties of the much higher energy cosmic muons

TABLE II. Sources of systematic uncertainties with the largest impact on $\Delta m_{32}^{2}$ and their effect on fitting the oscillation parameters for one standard deviation variations.

\begin{tabular}{lcc}
\hline \hline Uncertainty & $\begin{array}{c}\delta\left(\Delta m_{32}^{2}\right) \\
\left(10^{-3} \mathrm{eV}^{2}\right)\end{array}$ & $\delta\left(\sin ^{2} \theta_{23}\right)$ \\
\hline Atm. normalization CV $(15 \%)$ & 0.067 & 0.071 \\
Beam shower energy & 0.064 & 0.001 \\
Beam relative normalization $(1.6 \%)$ & 0.049 & 0.002 \\
$\mu^{ \pm}$energy (range 2\%, curv. 3\%) & 0.048 & 0.003 \\
Atm. normalization nonfid. $(25 \%)$ & 0.032 & 0.053 \\
Atm. $\bar{\nu}_{\mu} / \nu_{\mu}$ ratio CV $(10 \%)$ & 0.012 & 0.012 \\
\hline \hline
\end{tabular}




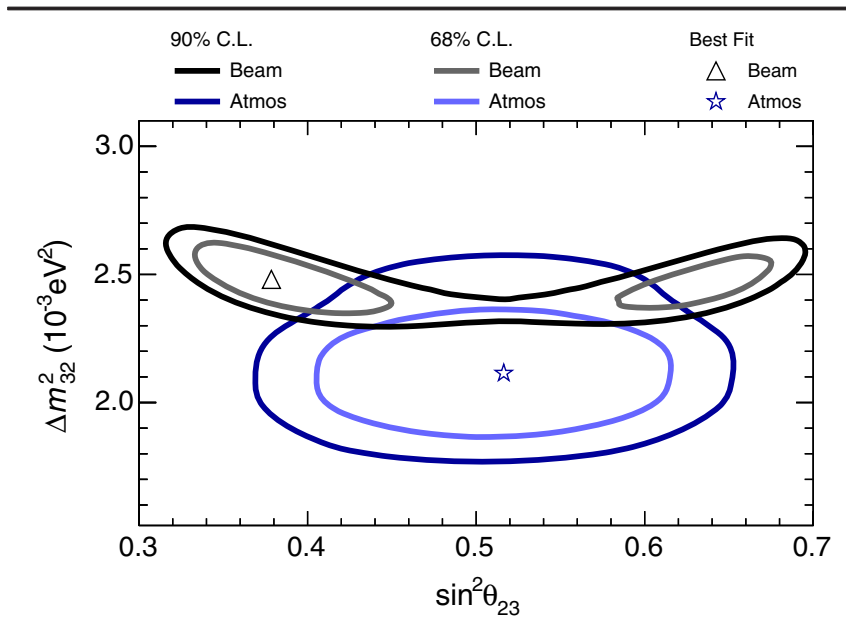

FIG. 3. Confidence limits on $\Delta m_{32}^{2}$ and $\sin ^{2} \theta_{23}$ in the normal hierarchy for MINOS and MINOS+ shown separately for the beam and atmospheric data. Contours are shown at 90\% C.L. and at $68 \%$ C.L. Best fit points are shown for the beam data (triangle) and for the atmospheric fit (star).

associated with this sample. Atmospheric CV events have an additional $10 \%$ uncertainty on the $\bar{\nu}_{\mu} / \nu_{\mu}$ ratio. These three uncertainties have the largest effects on measuring $\sin ^{2} \theta_{23}$ in the combined fit of beam and atmospheric data.

The final result sums the likelihood contributions coming separately from the combined $\nu_{\mu}$ disappearance and the MINOS $\nu_{e}$ appearance $[12,22]$ datasets. The $\nu_{\mu}$ disappearance and the $\nu_{e}$ appearance analyses use information from the Near Detector in independent ways. The $\nu_{\mu}$ analysis uses ND data to minimize uncertainties in the beam flux and neutrino cross section, while the $\nu_{e}$ analysis primarily uses ND data to estimate backgrounds. The dominant uncertainty is statistical; the systematic uncertainties in the two analyses are treated as uncorrelated.

Figure 2 shows the MC predictions for the best fit oscillation parameters for MINOS (hatched red) and MINOS+ (hatched blue). The combined MINOS and MINOS + MC spectrum is also shown (cyan). All MC samples with expected neutrino oscillations include the small contribution of background events from $\nu_{\tau}$ and $\bar{\nu}_{\tau}$ appearance. The oscillation parameters best-fit point obtained using only the MINOS+ neutrino beam data falls within the $1 \sigma$ contour from the previous MINOS measurement [4].

The $68 \%$ and $90 \%$ confidence level intervals in $\sin ^{2} \theta_{23}$ and $\Delta m_{32}^{2}$ parameter space for the normal hierarchy obtained for the beam and atmospheric samples separately are shown in Fig. 3. The confidence level intervals include the best fit points for the beam sample at $\sin ^{2} \theta_{23}=0.38$, $\Delta m_{32}^{2}=2.48 \times 10^{-3} \mathrm{eV}^{2}$ and for the atmospheric sample at $\sin ^{2} \theta_{23}=0.52, \Delta m_{32}^{2}=2.11 \times 10^{-3} \mathrm{eV}^{2}$. Studies of the compatibility of the atmospheric and beam results show a probability of $22 \%$ that they come from the same oscillation parameters.
TABLE III. The best fit values and confidence limits of the $\Delta m_{32}^{2}$ and $\sin ^{2} \theta_{23}$ parameters, calculated separately for the normal and inverted hierarchy. $\Delta m_{32}^{2}$ is reported in units of $10^{-3} \mathrm{eV}^{2}$.

\begin{tabular}{lccc}
\hline \hline Mass hierarchy & Parameter & Best fit & Confidence limits \\
\hline Normal & $\left|\Delta m_{32}^{2}\right|$ & 2.40 & $2.31-2.48(68 \%$ C.L.) \\
& $\sin ^{2} \theta_{23}$ & 0.43 & $0.37-0.65(90 \%$ C.L.) \\
Inverted & $\left|\Delta m_{32}^{2}\right|$ & 2.45 & $2.37-2.52(68 \%$ C.L.) \\
& $\sin ^{2} \theta_{23}$ & 0.42 & $0.37-0.65(90 \%$ C.L.) \\
\hline \hline
\end{tabular}

The oscillation parameters at the best fit point and confidence limits from the overall combined fit for the normal and inverted hierarchy are shown in Table III. Figure 4 shows the confidence limits on $\Delta m_{32}^{2}$ and $\sin ^{2} \theta_{23}$ and the likelihood profiles as functions of $\Delta m_{32}^{2}$ and $\sin ^{2} \theta_{23}$. The best fit point at $\Delta m_{32}^{2}=2.40_{-0.09}^{+0.08} \times$ $10^{-3} \mathrm{eV}^{2}$ and $\sin ^{2} \theta_{23}=0.43_{-0.04}^{+0.20}$ weakly favors nonmaximal mixing at $0.91 \sigma$ and the normal hierarchy at $0.45 \sigma$. This measurement of $\Delta m_{32}^{2}$ is competitive with that measured by $\mathrm{T} 2 \mathrm{~K}$ and $\mathrm{NO} \nu \mathrm{A}$. Differences between the best fit values of the parameters in Fig. 4 are providing added precision to global fits based on these 90\% C.L. $(1.6 \sigma)$ contours.
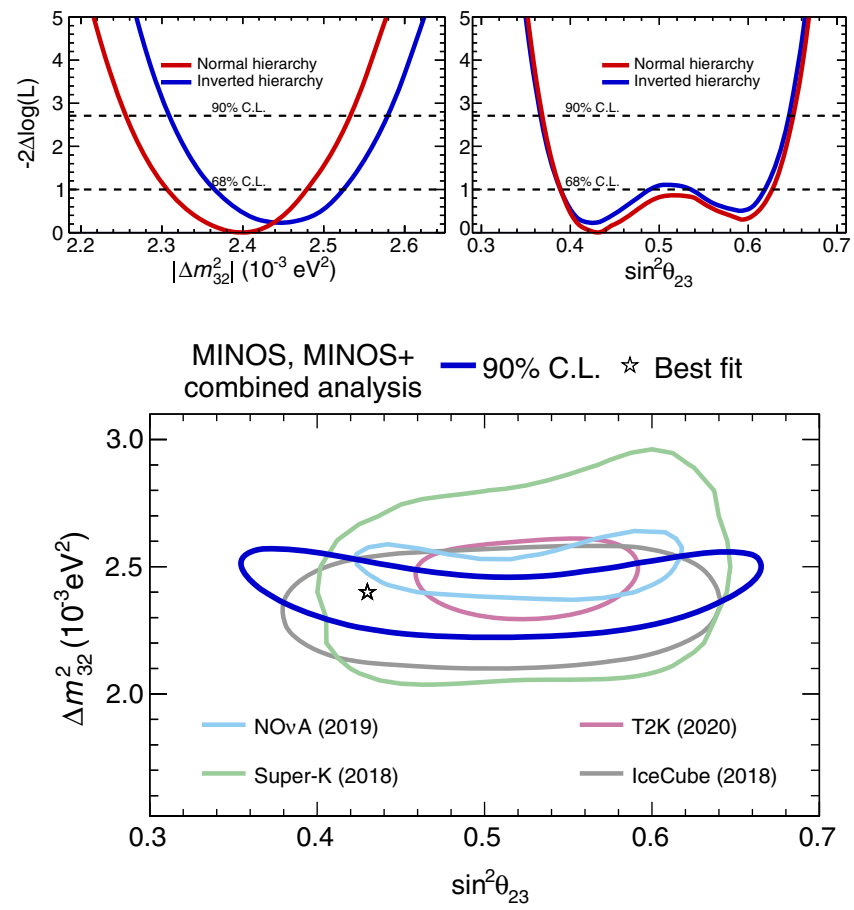

FIG. 4. The top figures show the 1D likelihood profiles as functions of $\Delta m_{32}^{2}$ and $\sin ^{2} \theta_{23}$ for each hierarchy. The bottom figure displays $90 \%$ confidence limits on $\Delta m_{32}^{2}$ and $\sin ^{2} \theta_{23}$ for the normal mass hierarchy, comparing MINOS+, IceCube, $\mathrm{NO} \nu \mathrm{A}$, Super-K, and T2K [5,7,8,11]. 
In summary, analysis of the $\nu_{\mu}$ disappearance and $\nu_{e}$ appearance samples from the complete beam and atmospheric datasets of the MINOS and MINOS+ run phases has been presented and provides new and competitive constraints on the oscillation parameters $\Delta m_{32}^{2}$ and $\sin ^{2} \theta_{23}$, weakly favors nonmaximal mixing, and exhibits octant degeneracy.

This work was supported by the U.S. DOE; the United Kingdom STFC, part of UKRI; the U.S. NSF; the State and University of Minnesota; and Brazil's FAPESP, CNPq, and CAPES. We are grateful to Fermilab and the Minnesota Department of Natural Resources and the personnel of the Soudan Laboratory. We thank the Texas Advanced Computing Center at The University of Texas at Austin for the provision of computing resources.

*Deceased.

${ }^{\dagger}$ Now at Physics Department, University of Wisconsin, Madison, Wisconsin 53706, USA.

[1] Y. Fukuda et al. (Super-Kamiokande Collaboration), Phys. Rev. Lett. 81, 1562 (1998).

[2] A. Gando et al. (KamLAND Collaboration), Phys. Rev. D 88, 033001 (2013).

[3] B. Aharmim et al. (SNO Collaboration), Phys. Rev. C 88, 025501 (2013).

[4] P. Adamson et al. (MINOS Collaboration), Phys. Rev. Lett. 112, 191801 (2014).

[5] M. G. Aartsen et al. (IceCube Collaboration), Phys. Rev. Lett. 120, 071801 (2018).

[6] K. Abe et al. (T2K Collaboration), Phys. Rev. Lett. 121, 171802 (2018).

[7] K. Abe et al. (T2K Collaboration), Nature (London) 580, 339 (2020); 583, E16 (2020).

[8] K. Abe et al. (Super-Kamiokande Collaboration), Phys. Rev. D 97, 072001 (2018).

[9] D. Adey et al. (Daya Bay Collaboration), Phys. Rev. Lett. 121, 241805 (2018).

[10] G. Bak et al. (RENO Collaboration), Phys. Rev. Lett. 121, 201801 (2018).

[11] M. A. Acero et al. (NOvA Collaboration), Phys. Rev. Lett. 123, 151803 (2019).

[12] P. Adamson et al. (MINOS Collaboration), Phys. Rev. Lett. 110, 251801 (2013).
[13] P. Adamson et al. (MINOS Collaboration), Phys. Rev. D 95, 012005 (2017).

[14] V. D. Barger, J. Learned, P. Lipari, M. Lusignoli, S. Pakvasa, and T. J. Weiler, Phys. Lett. B 462, 109 (1999).

[15] P. Adamson et al. (MINOS Collaboration), Phys. Rev. Lett. 106, 181801 (2011).

[16] J. A. B. Coelho, W. A. Mann, and S. S. Bashar, Phys. Rev. Lett. 118, 221801 (2017).

[17] P. Adamson et al. (MINOS+ Collaboration), Phys. Rev. Lett. 122, 091803 (2019).

[18] P. Adamson et al. (MINOS Collaboration), Phys. Rev. D 94, 111101 (2016).

[19] P. Adamson et al., Nucl. Instrum. Methods Phys. Res., Sect. A 806, 279 (2016).

[20] D. G. Michael et al. (MINOS Collaboration), Nucl. Instrum. Methods 596, 190 (2008).

[21] P. Adamson et al. (MINOS Collaboration), Phys. Rev. D 86, 052007 (2012).

[22] P. Adamson et al. (MINOS Collaboration), Phys. Rev. Lett. 110, 171801 (2013).

[23] P. Adamson et al. (MINOS Collaboration), Phys. Rev. D 77, 072002 (2008).

[24] M. Campanella, A. Ferrari, P. R. Sala, and S. Vanini, ATLAS internal note ATL-SOFT-99-004, 1999.

[25] G. D. Barr, T. K. Gaisser, P. Lipari, S. Robbins, and T. Stanev, Phys. Rev. D 70, 023006 (2004).

[26] H. Gallagher, Nucl. Phys. B, Proc. Suppl. 112, 188 (2002).

[27] D. Casper, Nucl. Phys. B, Proc. Suppl. 112, 161 (2002).

[28] Application Software Group, CERN Program Library Long Writeup W5013, CERN, 1994.

[29] C. Zeitnitz and T. A. Gabriel, Nucl. Instrum. Methods Phys. Res., Sect. A 349, 106 (1994).

[30] R. Ospanov, Ph. D. Thesis, University of Texas at Austin, 2008.

[31] J. Huang, Ph.D. Thesis, University of Texas at Austin, 2015.

[32] C. Backhouse, Ph. D. Thesis, University of Oxford, 2011.

[33] M. Strait, Ph. D. Thesis, University of Minnesota, 2010.

[34] J. D. Chapman, Ph.D. Thesis, University of Cambridge, 2007.

[35] B. P. Speakman, Ph.D. Thesis, University of Minnesota, 2007.

[36] A. J. Perch, Ph.D. Thesis, University College London, 2017.

[37] C. Patrignani et al. (Particle Data Group), Chin. Phys. C 40, 100001 (2016); see the review on neutrino mass, mixing, and oscillations.

[38] G. L. Fogli, E. Lisi, A. Marrone, D. Montanino, A. Palazzo, and A. M. Rotunno, Phys. Rev. D 86, 013012 (2012). 